Artigo Original

Original Article

\title{
Avaliação dos Fatores Reprodutivos em 117 Pacientes com Esclerose Sistêmica Forma Limitada e 72 Pacientes com Artrite Reumatóide $e^{(*)}$
}

\section{Comparison of Reproductive Factors in 117 Limited Scleroderma and 72 Rheumatoid Arthritis Patients}

\author{
Romy Beatriz Christmann de Souza ${ }^{(1)}$, Claudia Tereza Lobato Borges ${ }^{(2)}$, \\ Eduardo Ferreira Borba ${ }^{(3)}$, Eloísa Bonfá ${ }^{(4)}$
}

\section{RESUMO}

Recentemente, os fatores reprodutivos foram estudados na esclerose sistêmica (ES) difusa e a gravidez não parece ser um fator de risco para a doença. A validade desses achados precisava ser confirmada na forma limitada. Objetivo: comparar fatores reprodutivos em 372 gestações em ES na forma limitada (ESL) com 240 gestações em AR e seu risco em desenvolver ES. Métodos: cento e dezessete pacientes com ESL e 72 com artrite reumatóide (AR) consecutivas foram entrevistadas por meio de um questionário detalhado que avaliava história reprodutiva para estimar o risco em desenvolver ES. O odds ratio (OR) foi calculado usando como referência mulheres nulíparas e sem história de abortamento. Resultados: o diagnóstico de ESL e AR foi definido após as gestações na maioria das pacientes em ambas as doenças ( $97 \%$ vs. $90 \%$, respectivamente p>0,05). A média de idade ao diagnóstico (40,8 vs. 38,4 anos) e média de idade da primeira gravidez (22,7 vs. 23,5 anos) foi semelhante para as pacientes com ESL e com AR respectivamente, $p>0,05$. O número de gestações por paciente (3,1 vs. 3,3), porcentagem de pacientes que engravidaram pelo menos uma vez $(84,6 \%$ vs. $88,8 \%)$ e abortamento $(19,8 \%$ vs. $21,6 \%)$ foi similar em ambos os grupos, $\mathrm{p}>0,05$. Comparando as pacientes com ESL nulíparas com as multíparas, encontramos OR de 0,7, IC 95\% 0,3 - 0,7 para desenvolver ESL, e quando comparamos mulheres com e sem história de abortamento, OR foi de 0,9 IC 95\% 0,5 - 1,6. Além disso, o número de filhos não mostrou aumento no risco de desenvolver ESL quando comparado com nulíparas. Conclusões: estes dados sugerem que a história reprodutiva na ESL é similar à AR. Além disso, os dados predizem que a gravidez não parece causar aumento no risco nem

\begin{abstract}
Recently, reproductive factors have been studied in diffuse Systemic Sclerosis patients and pregnancy was not likely to be a risk factor for disease development. This findings need to be confirmed in the limited form of the disease. Objective: To compare reproductive factors in 372 limited systemic sclerosis (LSSC) and 240 rheumatoid arthritis (RA) pregnancies and their relative risk for developing LSSC. Methods: One hundred and seventeen LSSC and 72 RA consecutive female patients were interviewed with a detailed questionnaire to estimate the relative risk of developing LSSc given a relevant reproductive variable. The odds ratio (OR) was calculated using as reference nulliparous women and no history of miscarriage. Results: Diagnosis of LSSC and $R A$ was made after pregnancy for most patients of both diseases (97\% vs. $90 \%$, respectively, $p>0.05)$. A similar mean age at diagnosis (40.8 years old vs. 38.4 years old) and mean age of first pregnancy (22.7 years old vs. 23.5 years old) were noted in LSSC and RA $(p>0.05)$, respectively. The number of pregnancies/ patient (3.1 vs. 3.3), percentage of patients ever pregnant (84.6\% vs. $88.8 \%)$ and miscarriage (19.8\% vs. $21.6 \%)$ was similar in both groups ( $p>0.05)$. Compared to nulliparous women, parous LSSC had an OR of 0.7, 95\% CI 0.3-1.7 for developing LSSC and, for women who experienced a miscarriage, the [OR] was 0.9, 95\% CI 0.5-1.6, compared with those LSSc patients with no miscarriage. In addition to that, the number of pregnancies was not associated with an increased risk for LSSC when compared to nulliparous controls. Conclusions: These data suggest that the overall reproductive history of LSSC is comparable to RA. Moreover, it
\end{abstract}

* Trabalho realizado na Disciplina de Reumatologia da Faculdade de Medicina da Universidade de São Paulo (FMUSP), São Paulo, SP, Brasil. Recebido em 12/01/2005. Aprovado, após revisão, em 05/05/2005.

1. Pós-graduanda em Reumatologia na FMUSP.

2. Médica assistente do Serviço de Reumatologia do HC-FMUSP.

3. Médico assistente do Serviço de Reumatologia do HC-FMUSP.

4. Professora titular da Disciplina de Reumatologia da FMUSP.

Endereço para correspondência: Romy Beatriz Christmann de Souza. Faculdade de Medicina da Universidade de São Paulo (FMUSP). Disciplina de Reumatologia. Av. Dr. Arnaldo, 455, 3. ${ }^{\circ}$ andar, sala 3131, CEP 01246-903. São Paulo, SP, Brasil; e-mail: reumato@edu.usp.br 
ser um fator protetor no desenvolvimento de ESL quanto comparado com AR.

Palavras-chave: esclerose sistêmica, gravidez, microquimerismo, artrite reumatóide, fatores reprodutivos.

\section{INTRODUÇÃO}

A esclerose sistêmica (ES) é uma doença auto-imune que acomete principalmente mulheres na quarta e quinta décadas de vida. Essa descrição epidemiológica sugere que fatores hormonais ou reprodutivos poderiam estar associados na patogênese da doença ${ }^{(1)}$.

Estudos feitos nos anos 1990 detectaram a presença de pequenas quantidades de células fetais no sangue periférico na maioria das grávidas e a sua persistência durante vários anos após a gravidez, fenômeno conhecido como microquimerismo fetal ${ }^{(2-5)}$.

Atualmente, o microquimerismo fetal é umas das hipóteses propostas para explicar a patogênese da $\mathrm{ES}^{(6-10)}$. Nelson et al., em 1998, mostraram que a quantidade de células fetais nas mulheres com ES foi maior quando comparadas com mulheres sadias ${ }^{(7)}$. Além disso, a ES apresenta características similares à doença do enxerto contra o hospedeiro, uma desordem quimérica que ocorre em pacientes após transplante de medula óssea alogênico ${ }^{(11)}$.

Alguns estudos propõem-se a avaliar a história reprodutiva como fator de risco para ES. Launay et al., em 2001, avaliaram a vida reprodutiva de mulheres com ES e a compararam com a paridade e o sexo dos filhos nascidos antes da doença. Concluíram que o microquimerismo estaria possivelmente mais associado à forma limitada da ES e mais ao sexo feminino dos conceptos $^{(12)}$. Pisa et al., compararam dados da vida reprodutiva de mulheres com ES com pacientes ortopédicos de um hospital. Foram estudadas 44 pacientes na forma difusa da ES, quatro na forma limitada (ESL) e comparadas com 153 mulheres do grupo controle. Encontraram um risco relativo reduzido de desenvolver ES nas mulheres multíparas, questionando se a possibilidade da gravidez deve ser mesmo considerada como fator de risco para $\mathrm{ES}^{(13)}$.

Neste trabalho avaliamos a história reprodutiva de 117 pacientes com ESL que foram comparadas com 72 pacientes com diagnóstico de artrite reumatóide (AR). O grupo de AR foi escolhido como controle por ter características epidemiológicas semelhantes, acometendo mais mulheres, também após a vida reprodutiva. Além disso, estudos sugerem que o microquimerismo fetal teria efeito benéfico nessa doença ${ }^{(14,15)}$. indicates that pregnancy is unlikely to account for an increased risk or a protective effect to develop LSSc in comparison to RA.

Keywords: systemic sclerosis, pregnancy, microchimerism, rheumatoid arthritis, reproductive factors.

\section{PACIENTES E MÉTODOS}

Este estudo foi composto de 117 pacientes consecutivas do sexo feminino, com ESL que preenchiam os critérios de classificação da ACR (American College of Rheumatology) ${ }^{(16)}$ e o subtipo de doença foi definido na avaliação inicial de acordo com Le Roy ${ }^{(17)}$. Consideramos o início da doença a data em que a paciente apresentou o primeiro sintoma da ESL (fenômeno de Raynaud em todas).

Como controle foram estudadas 72 pacientes consecutivas, do sexo feminino com AR, conforme os critérios da $\mathrm{ACR}^{(18)}$, sendo considerado o início da doença a data em que recebeu o diagnóstico por um médico. Todas faziam o acompanhamento no Ambulatório de Reumatologia do Hospital das Clínicas da FMUSP.

Cada paciente foi entrevistada pelos autores do trabalho e seu prontuário revisado. A entrevista consistia de questões sobre a história reprodutiva, como: idade da primeira gestação ou abortamento (espontâneo ou induzido), número de gestações e abortamentos, sexo e seqüência dos filhos, intervalo entre a gestação e o desenvolvimento da doença (para ESL e AR).

Estatística - As características clínicas e história reprodutiva da ESL e da AR foram comparadas utilizando teste t-Student e qui-quadrado. Para estimar o risco da gestação em desenvolver a ESL, o odds ratio e seu intervalo de confiança de $95 \%$ foi utilizado. Na história reprodutiva, dados obtidos de mulheres nulíparas foram utilizados como referência. Dados das mulheres sem história de abortamento foram utilizados como referência para estimar o risco em desenvolver ESL em relação ao abortamento.

\section{RESULTADOS}

Os dados demográficos das 117 pacientes com ESL e 72 pacientes com AR revelaram que a média de idade atual (56,8 x 56,1 anos, $\mathrm{p}=0,73)$, média de idade por ocasião do diagnóstico $(40,8 \times 38,4$ anos, $p=0,22)$ e média de duração de doença $(15,9 \times 17,7$ anos, $\mathrm{p}=0,28)$ foram semelhantes nos dois grupos (Tabela 1).

Avaliamos 372 gestações de pacientes com ESL e 240 de pacientes com AR. A média do número de gestações e a 
idade da primeira gestação nos dois grupos foram similares. Ressaltamos que 105 (90,2\%) pacientes com ESL tiveram o seu diagnóstico após a primeira gestação ou abortamento, sendo que em um quinto destas a ESL foi diagnosticada no período de até dez anos. Estes dados foram semelhantes aos observados nas pacientes com AR, sendo que 91,3\% (65 pacientes) tiveram o diagnóstico após a primeira gestação, $\mathrm{p}>0,05$.

A Tabela 2 compara o grupo das pacientes com ESL. As nulíparas, comparadas com as que engravidaram, tiveram idade de início da doença mais precoce (32,4 vs. 42,3, respectivamente; $\mathrm{p}=0,005)$ e a duração da doença foi significativamente maior (21,3 vs. 14,9, respectivamente; $\mathrm{p}=0,003)$. Do grupo das mulheres com ESL, 74 gestações $(19,8 \%)$ evoluíram para abortamento e do total das 117 pacientes, 44 (37,6\%) tiveram pelo menos um abortamento. Pacientes com AR tiveram número semelhante de abortamentos (total de 52) sendo $21,6 \%$ de todas as 240 gestações. Trinta e uma (43\%) das 72 pacientes com AR tiveram pelo menos um abortamento. Todos os dados sem significância estatística.

A maioria dos abortamentos aconteceu antes do desenvolvimento da doença, sendo $88,6 \%$ na ESL e $87,1 \%$ da AR, p >0,05. Quando a primeira gestação das pacientes com ESL foi avaliada, 18 casos $(15,4 \%)$ tiveram abortamento. Da mesma forma, o total de abortamentos na primeira gravidez do grupo de AR foi de 15 (20,8\%) sem significância estatística. A média de idade das pacientes que tiveram como primeira gravidez o abortamento foi de 21,6 anos vs. 23,6 anos para ESL e AR, respectivamente, $p=0,31$.

A Tabela 3 mostra a correlação entre os fatores reprodutivos e o risco de desenvolver ESL. A distribuição de nulíparas entre os dois grupos foi semelhante, sendo 15,4\% (18 casos) e 11,1\% (8 casos) na ESL e AR, respectivamente $(\mathrm{p}>0,05)$. O risco de desenvolver ESL não foi maior nas que engravidaram quando comparadas com as nulíparas (OR de 0,7 [IC 95\% 0,3-1,7]), sugerindo que a gravidez não é um fator de risco para esta doença.

Da mesma forma, a história prévia de abortamento parece não ser um risco para o desenvolvimento da $\operatorname{ESL}(\mathrm{OR}=0,9$ [IC 95\% 0,5-1,6]). (Tabela 2).

A média de intervalo entre a primeira gestação de concepto do sexo masculino e o início da doença na ESL foi de 19,4 anos e de concepto do sexo feminino foi de 20,8 anos $(\mathrm{p}=0,64)$. Na AR, a média de intervalo entre a primeira gestação de concepto do sexo masculino e o início da doença foi de 15 anos e de concepto do sexo feminino de 17,6 anos $(p=0,51)$. Portanto, não houve diferença entre os dois grupos.
TABELA 1

Características ReProdutivas das Pacientes COM ESL E AR

\begin{tabular}{lccc}
\hline & ESL (n=117) & AR (n=72) & p* \\
\hline Idade atual (anos) & 56,8 & 56,1 & 0,73 \\
Idade diagnóstico (anos) & 40,8 & 38,4 & 0,22 \\
Duração da doença (anos) & 15,9 & 17,7 & 0,28 \\
Média de gestações & 3,1 & 3,3 & 0,70 \\
Idade da primeira gestação (anos) & 22,7 & 23,5 & 0,30 \\
Número de gestações & 372 & 240 & - \\
\hline
\end{tabular}

*Diferença significativa quando $\mathrm{p}<0,05$

TABELA 2

CARACTERÍsTICAS DAS PACIENTES COM ESL

\begin{tabular}{lccc}
\hline ES & Nulíparas & Engravidaram & * \\
\hline Idade de início da doença & 32,4 anos & 42,3 anos & 0,005 \\
Duração da doença & 21,3 anos & 14,9 anos & 0,003 \\
\hline
\end{tabular}

*Diferença significativa quando $p<0,05$

TABELA 3

RAZÃO DE CHANCES DE DESENVOLVIMENTO DE ESL EM RELAÇÃO AOS FATORES REPRODUTIVOS. GRUPO CONTROLE $=$ AR

\begin{tabular}{lcccc}
\hline & ESL & AR & OR & $95 \%$ IC \\
\hline Gravidez-n(\%) & & & & \\
$\quad$ Não* & $18(15)$ & $8(11)$ & 1,0 & \\
$\quad$ Sim & $99(85)$ & $64(89)$ & 0,7 & $0,3-1,7$ \\
Abortamentos-n(\%) & & & & \\
$\quad$ Não* & $55(56)$ & $33(46)$ & 1,0 & \\
$\quad$ Sim & $44(44)$ & $31(54)$ & 0,9 & $0,5-1,6$ \\
Número de filhos & & & & \\
0* & $18(15)$ & $8(11)$ & 1,0 & \\
1 & $11(9)$ & $11(15)$ & 0,4 & $0,1-1,4$ \\
2 & $22(19)$ & $10(14)$ & 1,0 & $0,3-3,0$ \\
3 & $21(18)$ & $17(24)$ & 0,5 & $0,2-1,6$ \\
4 & $21(18)$ & $11(15)$ & 0,8 & $0,3-2,6$ \\
$>4$ & $24(21)$ & $15(21)$ & 0,7 & $0,2-2,0$ \\
\hline
\end{tabular}

OR (IC 95\%). * valor de referência

Não houve uma diferença importante no risco em relação ao número de filhos. Houve, porém, uma tendência de menor risco naquelas mulheres que tiveram um ou mais filhos. 


\section{DISCUSSÃo}

Atualmente, o microquimerismo fetal tem sido proposto como um fator importante na patogênese da ES. Investigando esta hipótese, alguns trabalhos visam mostrar uma evidência da relação da gravidez com ES. Um trabalho recente de um grupo italiano, avaliando principalmente pacientes com ES com a forma difusa da doença ${ }^{(13)}$, mostrou que as multíparas apresentam um risco reduzido de ES quando comparadas com nulíparas. Nesse mesmo estudo, identificaram uma associação inversa entre o risco de ES e o número de filhos, o que levou os autores a concluírem que a gravidez por si só não é um fator de risco para a ES na forma difusa.

Nosso estudo limitou-se à ES na forma limitada e também mostrou que pacientes com ESL e multíparas, quando comparadas com nulíparas, apresentam um risco semelhante de desenvolver ES. Além disso, mulheres que tiveram pelo menos um abortamento, quando comparadas com as que nunca abortaram, também não apresentaram risco maior para ES. Portanto, conforme sugerido pelo grupo italiano na forma difusa, a gravidez e o abortamento não parecem contribuir para o aumento do risco para o desenvolvimento da ES na forma limitada.

Quanto à fertilidade, nosso estudo mostrou que das 117 pacientes com ESL entrevistadas, 85\% engravidaram com uma média de 3,3 gestações por paciente, índices semelhantes ao da população normal. Da mesma forma, na AR, como já mostrado em outros estudos, a fertilidade é igual à da população normal ${ }^{(19)}$. Steen e Medsger, em 1999, mostraram que $21 \%$ das mulheres com ES (45\% delas com forma difusa), diagnosticadas antes dos 45 anos, nunca engravidaram. Excluindo-se fatores como vida sexual ativa e aquelas que nunca tentaram engravidar, porém, a infertilidade não ocorre mais freqüentemente em pacientes com ES quando comparadas com controle ${ }^{(20)}$.

Launay et al., em 2001, avaliaram 100 mulheres com ES e evidenciaram que tanto na forma difusa quanto na limitada, a doença tem início em idade semelhante. Entretanto, separando-se o grupo em multíparas e nulíparas, as multíparas com a forma limitada tiveram início mais precoce de doença $(35,8 \pm 14,4 \text { anos })^{(12)}$.

Interessantemente, em nosso estudo, entre as pacientes com ESL a idade de início da doença foi mais precoce nas nulíparas. Além disso, a duração da doença neste grupo foi maior $(21,3$ vs. 14,9 anos $\mathrm{p}<0,05)$. Uma explicação para a nuliparidade seria a opção de não engravidar ou aconselhamento para não engravidar, motivo já comprovado em estudos anteriores ${ }^{(20)}$.
Nossos dados mostraram que o grupo de ESL apresentou uma freqüência mais elevada de abortamento quando comparado com outros estudos. Entretanto, quando comparado com o grupo controle composto por pacientes com AR, esta porcentagem foi semelhante. Em trabalhos mais antigos, a freqüência de abortamentos na ES era maior, porém existia pouca concordância de resultados entre centros e a maioria destes estudos não era controlada. Num estudo grego, o abortamento ocorreu em $22 \%$ dos pacientes com ES, comparados com $12 \%$ do grupo controle sadio. Apesar da diferença significante, o valor encontrado na ES é semelhante ao da população geral ${ }^{(19)}$. Silman e Black encontraram nas pacientes com ES 29\% de abortamentos, número significativamente maior que o grupo controle normal estudado $(17 \%)^{(21)}$.

Mais recentemente, alguns estudos mostraram que a freqüência de abortamento não era maior na ES. Steen e Medsger, em 1999, avaliaram 214 pacientes com ES e encontraram uma freqüência de abortamento de $22 \%$ a $26 \%$, semelhante ao grupo controle de 167 pacientes com AR e 105 controles normais ${ }^{(20)}$. Silman e Black, em um estudo caso-controle, mostraram freqüências similares de abortamentos nas pacientes com ES e nos controles com fenômeno de Raynaud primário e normais $\left.{ }^{(21}\right)$.

Quanto à influência do sexo dos filhos, um estudo mostrou que o intervalo entre o primeiro concepto e o início da ES foi menor quando este foi do sexo feminino. Aventaram, então, a possibilidade de que um filho do sexo masculino poderia induzir uma resposta auto-imune materna mais eficaz, provocando a eliminação de suas células fetais da circulação materna. Por outro lado, um concepto do sexo feminino, por ter uma maior semelhança com as células maternas (ausência do cromossomo Y), poderia então dificultar o seu reconhecimento como antígeno externo e induzir uma tolerância imunológica, provocando a doença ${ }^{(22)}$. Nosso trabalho, entretanto, não conseguiu mostrar diferenças no tempo de início da doença com relação ao sexo do primeiro filho em pacientes com ES forma limitada.

Finalmente, observamos que o número de filhos não influenciou no desenvolvimento de ESL, mostrando inclusive uma tendência de menor risco quando na presença de mais de um filho.

Em resumo, mostramos que na ESL a gravidez e o abortamento não são fatores de risco para o desenvolvimento da doença.

Entendemos que outros estudos epidemiológicos serão necessários para esclarecer a real influência dos fatores reprodutivos na patogênese da ES. 


\section{REFERÊNCIAS}

1. Maureen DM: Epidemiology of Systemic Sclerosis and related diseases. Curr Op Rheumatol 9: 557-61, 1997.

2. Bianchi DW: Prenatal diagnosis by analysis of fetal cells in maternal blood. J Pediatr 127: 847-56, 1995.

3. Thomas MR, Williamson R: Y chromosome sequence DNA amplified from peripheral blood of women in early pregnancy. Lancet 343: 413-4, 1994.

4. Lo YMD, Tein MSC, Lau TK: Quantitative analysis of fetal DNA in maternal plasma and serum: implications for noninvasive prenatal diagnosis. Am J Hum Genet 62: 768-75, 1998.

5. Carol MA, Bruce S, Jimenez SA: Identification of fetal DNA and cells in skin lesions from women with Systemic Sclerosis. N Engl J Med 338: 1186-91, 1998

6. Nelson JL: Microchimerism and pathogenesis of Systemic Sclerosis. Curr Op Rheumatol 10: 546-71, 1998.

7. Nelson JL, Daniel EF, Maloney S, et al: Microchimerism and HLA - compatible relationships of pregnancy in Scleroderma. Lancet 351: 559-62, 1998.

8. Carol MA, Lori AC, Jimenez SA: Detection of cellular microchimerism of male or female origin in Systemic Sclerosis patients by polymerase chain reaction analysis of HLA - Cw antigens. Arthritis Rheum 43: 1062-7, 2000.

9. Lambert NC, Stevens AM, Tylee TS, Erickson TD, Furst DE, Nelson JL: From the simple detection of microchimerism in patients with autoimmune diseases to its implication in pathogenesis. Ann N Y Acad Sci 945: 164-71, 2001.

10. Kirby LJ, Nelson JL, Furst DE, et al: Fetal cell microchimerism in tissue from multiple sites in women with Systemic Sclerosis. Arthritis Rheum 44: 1848-54, 2001.

11. Bruce D, Claman HN: Chronic Graft-versus-Host Disease (GVHD) as a model for Scleroderma. Cell Immunol 77: 1-12, 1983.
12. Launay D, Hebbar M, Hatron P-Y, et al: Relationship between parity and clinical and biological features in patients with Systemic Sclerosis. J Rheumatol 28: 509-13, 2001.

13. Pisa FE, Bovenzi M, Romeu L, et al: Reproductive factors and the risk of Scleroderma. An Italian case-control study. Arthritis Rheum 46: 451-56, 2002.

14. Nelson JL, Kathleen A, Smith AG, Nisperos BB, Branchaud AM, Hansen JA: Maternal-fetal disparity in HLA Class II alloantigens and the pregnancy-induce amelioration of Rheumatoid Arthritis. N Engl J Med 329: 466-71, 1993.

15. Nelson JL: Maternal-fetal immunology and autoimmune disease: is some autoimmune disease auto-alloimmune or allo-autoimmune? Arthritis Rheum 39: 191-4, 1996.

16. Masi AT, Rodnan GP, Medsger T, et al: Preliminary criteria for the classification of systemic sclerosis. Arthritis Rheum 23: 581-90, 1980.

17. LeRoy EC, Black C, Fleischmajer R, et al: Scleroderma (systemic sclerosis): Classification, subsets and pathogenesis. J Rheumatol 15: 202-5, 1988.

18. Arnett FC, Edworthy SM, Bloch DA, et al: The American Rheumatism Association 1987 revised criteria for the classification of rheumatoid arthritis. Arthritis Rheum 31: 315-24, 1988.

19. Siamopoulou-M A: Outcome of pregnancy in patients with autoimmune rheumatic disease before the disease onset. Ann Rheum Dis 47: 982-7, 1988.

20. Steen VD, Medsger TA Jr: Fertility and pregnancy outcome in women with Systemic Sclerosis. Arthritis Rheum 42: 763-68, 1999.

21. Silman AJ, Black C: Increased incidence of spontaneous abortion and infertility in women with Scleroderma before disease onset: a controlled study. Ann Rheum Dis 47: 441-4, 1988.

22. Scaletti C, Vultaggio A, Bonifacio S, et al: Th2-oriented profile of male offspring $\mathrm{T}$ cells present in women with Systemic Sclerosis and reactive with maternal Major Histocompatibility Complex Antigens. Arthritis Rheum 46: 445-50, 2002. 